\title{
More (Good) Psychiatry
}

\author{
Howard A. Chang ${ }^{1}$ \\ Received: 11 December 2021 / Accepted: 18 February 2022 \\ (C) Academic Psychiatry 2022
}

During my clerkships as a medical student, I discovered an unusual similarity among internists, surgeons, pediatricians, obstetricians, and gynecologists: their reply when I informed them that I was interested in psychiatry.

"We need more good psychiatrists."

I heard this statement often, and initially it baffled me. Don't we need more good doctors in all areas of medicine?

But what often followed made it clear that these physicians paid particular attention to the existing state of mental healthcare because it greatly impacted their own patients. Frequently, they offered some personal or professional insight into psychiatry. Sometimes they shared how their patients' psychiatric comorbidities impeded treatment of their medical problems - for instance, the forgetfulness in depression that reduced medication adherence, the substance use that damaged patients' health, or the personality disorder that garnered physician bias. Recent trends in medicine corroborate the necessity of addressing mental health needs among patients everywhere, not just psychiatry. In obstetrics and gynecology, for example, evolving recognition of the complexities of psychiatric care during reproductive life events prompted the burgeoning of reproductive psychiatry as a subspecialty. Psycho-oncology is another relatively young subspecialty that emerged to better understand and meet the psychosocial needs of patients with cancer as survivorship continues to lengthen.

Pediatricians especially voiced the need for more psychiatrists. Children and adolescents would be among the greatest beneficiaries of improved access to psychiatric care. In the United States, there are approximately 11 child and adolescent psychiatrists per 100,000 youth [1]. This shortage exists amidst a mental health crisis afflicting people ages 10 to 24 ,

Howard A. Chang

hchang68@jhmi.edu

1 Johns Hopkins University School of Medicine, Baltimore, MD, USA for whom the rate of suicide has increased by $57 \%$ between 2007 and 2018 [2].

Surgeons also acknowledged their patients' mental health needs. One bariatric surgeon remarked that the operation is usually unsuccessful in patients who are unwilling to optimize their diet and exercise. Depression, common in the postoperative setting, makes it especially challenging to perform healthy behaviors and thus predicts worse weight loss outcomes following bariatric surgery. Broadly speaking, serious mental illness increases the odds of postoperative complications and longer hospital stays [3].

Over $50 \%$ of Americans want access to mental healthcare but many face barriers such as high costs, inadequate insurance coverage, and limited options [4]. Data like these demonstrate that increasing access to affordable psychiatric care is vital to ameliorating today's mental health crisis. Much of the work needed to achieve this goal requires systemic changes. These include, among others, government funding to increase the number of psychiatry residency positions, attractive programs that mobilize students to practice in under-resourced settings, insurance plans that incentivize hospitals to hire more mental health practitioners, and healthcare reforms that improve access to mental healthcare for those who cannot afford to pay out-of-pocket.

But generating more psychiatrists, while necessary, is insufficient. Indeed, the perspective expressed by many of the physicians I interacted with was not simply that we need more psychiatrists. It was that we need more good psychiatrists.

Principally, most suggested that "good" psychiatry involved formal psychotherapy. Yet between 2010 and 2016, $53 \%$ of psychiatrists in the United States did not provide psychotherapy services, and patients who received these services were more likely to be socioeconomically advantaged [5]. While psychotherapy is crucial to care for many patients, the practice settings of many psychiatrists may not permit them to offer dedicated psychotherapy sessions. This is why they often take care of patients in teams alongside licensed therapists who can do this important work. What all psychiatrists should offer patients is supportive psychotherapy, which involves 
building therapeutic alliance to help patients deal with psychosocial and emotional problems through empathic listening, encouragement, validation, comfort, reframing, advice, and other interpersonal techniques. Supportive psychotherapy does not require any specific setting and can be reasonably done in most patient encounters. Good psychiatrists, therefore, do not need to offer formal psychotherapy, but all should be willing and effective at using their words as medicine for their patients in a variety of clinical contexts. This is in stark contrast to the stereotypical 10-minute "med checks" that discredit the field to the public, or worse, the reduction of patients to psychopathologies rather than human beings deserving of respect.

It struck me how readily and uniformly physicians offered recommendations to a medical specialty they did not themselves practice. I could not recall ever hearing a noncardiologist argue that we need better medications in cardiology or a non-pulmonologist remark that we need greater diagnostic acumen in pulmonology. Psychiatry has always seemed to me most vulnerable to judgment — both good and bad - by spectators both within and outside of medicine. As a medical student interested in psychiatry, I view these sentiments as a heartfelt encouragement to, and validation of, a field increasingly perceived to be vital not only within medicine but throughout society.

How then can I become a good psychiatrist?

I have gleaned that a good psychiatrist fundamentally sees and cares about patients with mental illness as dignified human beings, not broken brains. The good psychiatrist knows and treats the person in order to treat the disease.

I can strive to practice good psychiatry by exploring my patients' interests and hobbies, not simply their signs and symptoms. I can ask them what their illness feels like and means to them, rather than assuming that I already know from referencing the Diagnostic and Statistical Manual of Mental Disorders. I can read about the lived experiences of those with mental illness, practice greater empathy, and engage with families and caregivers. I can emulate excellent psychiatrists in how they practice supportive psychotherapy. I can also meditate daily on my values and care for my own mental health to improve my ability to be fully present with patients.

These are not minor adjustments, mere add-ons to the "real" systemic changes that will rarefy the statement "We need more good psychiatrists." Rather, this is the central work I can begin to do now, as a trainee, to dignify the exhortation that a watching world has given me.

\section{Declarations}

Disclosures The author states that there is no conflict of interest.

\section{References}

1. American Academy of Child and Adolescent Psychiatrists. AACAP Work Force Fact Sheet. 2018. Available from: https://www.aacap. org/App_Themes/AACAP/docs/resources_for_primary_care/ workforce_issues/workforce_factsheet_updated_2018.pdf. Accessed 11 December 2021.

2. Curtin SC. State suicide rates among adolescents and young adults aged 10-24: United States, 2000-2018. Natl Vital Stat Rep. 2020;69(11):1-9.

3. McBride KE, Solomon MJ, Bannon PG, Glozier N, Steffens D. Surgical outcomes for people with serious mental illness are poorer than for other patients: a systematic review and meta-analysis. Med J Aust. 2021;214(8):379-85.

4. Cohen Veterans Network. National Council for Behavioral Health. America's Mental Health 2018. 10 October 2018. Available from: https://www.cohenveteransnetwork.org/wp-content/uploads/2018/ 10/Research-Summary-10-10-2018.pdf. Accessed 11 December 2021.

5. Tadmon D, Olfson M. Trends in outpatient psychotherapy provision by U.S. psychiatrists: 1996-2016. Am J Psychiatry. 2021. Online ahead of print. https://doi.org/10.1176/appi.ajp.2021.21040338.

Publisher's Note Springer Nature remains neutral with regard to jurisdictional claims in published maps and institutional affiliations. 